\title{
Determination of Malmquist Bias and Selection Effects from Monte-Carlo Simulations
}

\author{
Wolfram Freudling \\ Space Telescope-European Coordinating Facility, European Southern Observatory, Karl \\ Schwarzschild Str. 2, D-85748 Garching b. München, Germany \\ Luiz N. Da Costa \\ Institut d'Astrophysique, 98 bis Boulevard Arago, F75014, Paris, France and Observatorio \\ Nacional, Rua Gen. J. Cristino 77, São Cristovão, Rio de Janeiro, Brazil \\ Gary Wegner \\ Dept. of Physics and Astronomy, 6127 Wilder Lab., Dartmouth College, Hanover, NH 03755 \\ and Astronomisches Institut, Ruhr-Universität Bochum, D-44780 Bochum, Germany \\ Riccardo Giovanelli and Martha P. Haynes \\ Center for Radiophysics and Space Research and National Astronomy and Ionosphere Center ${ }^{1}$, \\ Cornell University, Ithaca, NY 14953 \\ John J. Salzer
Dept. of Astronomy, Wesleyan University, Middletown, CT 06457
}

Accepted for publication in The Astronomical Journal

\footnotetext{
${ }^{1}$ The National Astronomy and Ionosphere Center is operated by Cornell University under a cooperative agreement with the National Science Foundation.
} 


\begin{abstract}
Maps of the peculiar velocity field derived from distance relations are affected by Malmquist type bias and selection effects. Because of the large number of interdependent effects, they are in most cases difficult to treat analytically. Monte Carlo simulations are used to understand and evaluate these effects. In these simulations the "true" spatial distribution and relevant properties of galaxies as well as selection effects and observational uncertainties are realistically modeled. The results of the simulation can directly be applied to correct observed peculiar velocity maps. The simulation is used to investigate biases in samples of measured peculiar velocities by Lynden-Bell et al. (1988), Willick (1990) and the new sample of spiral galaxies by Haynes et al. (1993). Based on the results obtained from the application of our method to toy models we find that the method is a useful tool to estimate the bias induced both by inhomogeneities and selection effects. This is a crucial step for the analysis of the Haynes et al. sample which was selected with a redshift dependent criterion.
\end{abstract}

Subject headings: large scale structure of the universe - cosmology: observations - galaxies: clustering - galaxies: distances and redshifts 


\section{Introduction}

Maps of the peculiar velocity field of galaxies are a powerful tool to test scenarios for formation of large scale structure. However, the interpretation of such maps must take into account biases introduced by the coupling of errors in the distance measurements and the spatial distribution of galaxies and/or selection effects in the samples considered. Such biases in the derived distances are present even if the underlying distance relation is exactly known (which we will assume throughout this paper). A discussion of how a biasfree distance relation can be derived from data will be presented by Giovanelli et al. (1995). Biases originating from the spatial distribution are usually referred to as Malmquist bias and to correct for it requires a detailed knowledge of the underlying distribution of galaxies from which the sample is drawn. Biases introduced by the selection of the sample, which we will call selection biases throughout this paper, are caused by the correlation of the parameters used to select the sample with parameters used to estimate distances. In order to correct for such biases, the distribution function describing relevant galaxy properties is needed, as well as the detailed criteria adopted in selecting the sample being considered.

A number of correction schemes for Malmquist bias have been suggested by different workers (e.g., Feast 1987, Lynden-Bell et al. 1988; Landy \& Szalay 1992; Willick 1994). A common feature of all these approaches is that they require a priori knowledge of the distribution of galaxies in space, which has to be either assumed (e.g. Lynden-Bell et al. 1988) or, in the non-trivial case of an inhomogeneous distribution, derived from the data before the correction for the bias can be made. Different methods for estimating the required density distribution have been considered. Dekel (1993) built a treatment of the inhomogeneous Malmquist bias into their reconstruction of the density field, assuming that the distribution of galaxies traces the underlying matter distribution derived from peculiar velocities. Another approach utilizes the self-consistently reconstructed density field derived from redshift surveys (e.g., Dekel 1994, Hudson 1994).

Biases resulting from selection effects can in some situations be either comparable or larger than the Malmquist bias. To correct for selection biases, detailed knowledge of the selection process is necessary. However, even if this information is available it is difficult to derive simple expressions for situations where the parameter used to select the sample is not identical but correlates to one or several parameters used to derive distances. This is especially true when a complex set of different selection criteria is used to select the sample. For example, samples where the selection depends explicitly on the redshift, like the recently completed survey of peculiar velocities of Sc galaxies (Haynes et al. 1993, hereafter referred to as the the Sc sample), are particularly difficult to correct for this kind of bias.

The biases mentioned above are strongly related to the scatter of the particular distance relation used. For example, the scatter in the Tully-Fisher (TF) relation depends on the line widths of the galaxies in the sense that the scatter is large for galaxies of lower luminosity (Giovanelli et al. 1995). Such an effect would be difficult to treat analytically. This fact and the unique selection criteria of the Sc sample have motivated us to address the question of biases, both Malmquist and selection biases, using a Monte-Carlo (MC) 
technique.

MC simulations of Malmquist bias and/or selection effects have been used in the past by a number of workers (e.g. Landy \& Szalay 1992, Teerikorpi 1993, Willick 1994), mainly for the purpose of testing analytic corrections. In contrast, here the MC technique is used to determine the bias at each point in space to correct measured peculiar velocity fields. The essence of our method is to simulate galaxy catalogs using the spatial and parametric distribution derived from observations, and select redshift-distance samples similar to those currently available. The strength of the method is that all relevant effects can be incorporated to predict the bias without the need for any simplifying assumptions.

In section 2 we review analytical corrections for Malmquist bias which are used to test our MC results in simple cases. In section 3 a detailed description of the technique is presented. In section 4 we compare the bias derived from our MC simulations with analytical corrections and in section 5 we derive the bias field for observational samples. A brief summary is presented in section 6 .

\section{Biases in the Peculiar Velocity Field}

Different analytical expressions to correct for the Malmquist and selection type bias can be found in the literature (see e.g. Willick 1994; and references therein). These expressions depend critically on the properties of the scatter in the distance relation used. For example, to discuss the bias on distances estimated from a $\mathrm{TF}$ relation given by

$$
I=a_{T F} \log w+b_{T F}
$$

one can distinguish between the 'direct' and 'inverse' TF relation. In equation (1) $I$ is the absolute I-band magnitude and $w$ is the corrected HI line width. The 'direct' relation (called 'forward' relation by Willick 1994) is derived from a least-squares fit of $I$ as a function of $\log w$, while the 'inverse' relation is obtained from a least-squares fit of $\log w$ as a function of $I$. Independent of the distribution of the scatter about the line defined by equation (11), a normal distribution in $\log w$ can be used as an approximation for the inverse relation, while a normal distribution in $I$ will be a better approximation for the direct relation. Although in this paper we focus our attention on the TF relation, our conclusions apply equally well to the $D_{n}-\sigma$ relation.

A raw estimate of the distance $d_{\text {raw }}$ from a measured magnitude $m_{I}$ and line width $w$

$$
\log d_{\text {raw }}=0.2\left(m_{I}-a_{T F} \log w-b_{T F}\right)-3
$$

is a biased estimate of the distance. The most general expression to correct $d_{\text {raw }}$ obtained with the direct TF relation under the assumption that the scatter is normal in magnitudes is

$$
d_{c o r}=\frac{\int_{0}^{\infty} r^{3} n(r) \exp \left(-\frac{\left[\ln \left(r / d_{\text {raw }}\right)\right]^{2}}{2 \Delta^{2}}\right) d r}{\int_{0}^{\infty} r^{2} n(r) \exp \left(-\frac{\left[\ln \left(r / d_{\text {raw }}\right)\right]^{2}}{2 \Delta^{2}}\right) d r}
$$

where $d_{c o r}$ is the corrected estimated distance, $\Delta^{2}$ is the variance in the logarithm of the distance, and $n(r)$ is proportional to the space distribution of galaxies along the line of sight (e.g., Willick, 1994).

Landy \& Szalay (1992) have suggested a different expression given by

$$
d_{c o r}=d_{\text {raw }} \exp \left(\frac{\Delta^{2}}{2} \frac{h\left[\ln d_{\text {raw }}+\Delta^{2}\right]}{h\left[\ln d_{\text {raw }}\right]}\right)
$$


where $h(d)$ is the observed distribution of galaxies along the line of sight. As pointed out by Teerikorpi (1993), this expression is applicable when distances have been derived using the inverse TF relation.

Both expression (3) and (4) can be easily applied for the homogeneous case. They can also be easily applied to areas of the sky where the distribution of observed galaxies can be adequately described by one dimensional functions $h(d)$ or $n(r)$. However, if larger regions of the sky are considered, variation with the direction in the sky must be taken into account. For example, estimates for $n(r)$ can be obtained from redshift surveys taking into account expected peculiar velocities (Hudson 1994).

Willick (1991) also presented a formal framework to correct for selection biases which is similar to the corrections for Malmquist bias given in equation (3). Unfortunately, his expressions cannot directly be applied to a sample selected as the Sc sample.

In the analysis of the Sc sample we expect significant contributions from both Malmquist bias and selection effects due to the selection criteria adopted. This has prompted us to develop the MC approach presented below. In section 4 we compare the biases of the analytical expressions given in this section to results from our MC technique discussed below.

\section{The Monte Carlo Simulation}

\subsection{Method}

The ultimate goal of the method is to create simulated redshift - distance surveys with the same properties and thus the same biases as in existing observed samples. To achieve this, we have to simulate properties like the spatial distribution, magnitude $-\mathrm{di}$ - ameter function, color relations, distance relations and their respective scatter properties. In addition, the selection criteria and completeness of the observed samples under consideration have to be taken into account.

For the spatial distribution of galaxies, we use the density field derived from redshift surveys corrected for peculiar velocities (see below). The derived density field depends on the assumed $\beta=\Omega / b^{0.6}$, where $\Omega$ is the density parameter and $b$ is the linear biasing of galaxies relative to matter. In this paper, we assume that $\beta=1$. It should be emphasized that the density field and therefore the simulation is only slightly model dependent.

We use this density field to create a large number of artificial galaxies, to which we assign properties similar to those of the observed population of galaxies. We then simulate the selection of catalogs from which observational samples are drawn. Subsequently, the observational sample is drawn from the simulated catalog, following the same selection criteria used for existing surveys, including any known incompleteness of the observational sample. Although below we assume that this incompleteness depends only on the magnitudes, this can be easily generalized to include incompleteness as a function of direction in the sky. Finally, estimated distances are derived from the assumed distance relation.

The final sample, which contains both the true and the estimated distances, can now be used to compute the bias field in estimated distance or redshift space. For each artificial galaxy, the bias is computed by subtracting from the estimated distance the known "true" distance of galaxies. This bias affects not only the position of the galaxies but also its estimated peculiar velocity which can also be cor- 
rected using the same bias correction. Since the artificial catalogs of galaxies are large, the uncertainty in the determination of the biases can be reduced by binning them on a grid either in redshift or in estimated distance space. This average bias can then be applied to observed samples selected like the simulation. The raw estimated distance can be used to select the appropriate bias from the simulation and the estimated distance can be corrected for it. Peculiar velocities are subsequently computed from the bias corrected distances.

A summary of the items and parameters that represent the built-in assumptions for the estimate of biases are the parameters describing the luminosity function, the TF relation and the color-line width relation, the density distribution of galaxies, the peculiar velocities of galaxies, and the detailed selection and completeness of the observed sample. The main advantage of this approach is the flexibility it provides to evaluate a variety of effects and their impact on the estimated distances, including the scatter in the various relations used, the properties of the distance relations and the selection and incompleteness of observational samples.

\subsection{Procedure}

\subsubsection{Creating the 3d Galaxy Distribution}

To prepare the MC simulation, either a homogeneous distribution of galaxies or a distribution modeled after the local universe is used to derive a density field in real space. For the latter, the Optical-IRAS model without bias of Freudling et al. (1994) is used. This model uses the redshift distribution of optically selected galaxies in the galactic caps $\left(b<-30^{\circ}\right.$ or $\left.b>40^{\circ}\right)$, the 1.9Jy IRAS sample in re- gions $|b|>5^{\circ}$, and interpolates the density field over the galactic plane $\left(|b|<5^{\circ}\right)$. The positions of the galaxies are computed iteratively from the redshifts, using the linear approximation to estimate the peculiar velocity of each galaxy in each iteration. The final output is a self-consistent density field, which is stored on a Cartesian grid of $500 \mathrm{~km} \mathrm{~s}^{-1}$ cell size. This final density field is shown by Willmer et al. (1995).

The first step in the MC simulation is to create a list of galaxy coordinates which distributes galaxies randomly but with density fluctuations identical to those in the OpticalIRAS model. This is accomplished by interpreting the local density of the model as the probability $p$ to draw a galaxy at that location. The $\mathrm{x}, \mathrm{y}$ and $\mathrm{z}$ components of the position of potential galaxies are randomly drawn. The object is then rejected with a frequency proportional to $1-p$.

Finally, each galaxy is given a redshift computed from its distance, the velocity field of the Optical-IRAS model, and an assumed Gaussian random component. The distances are assumed to be proportional to the unperturbed redshift in the CMB restframe and are expressed in $\mathrm{km} / \mathrm{sec}$ throughout this paper. For computational efficiency, the peculiar velocity vector is computed only once for each cell on the density mesh, and all galaxies within each cell are given the same peculiar velocity field. Subsequently, a Gaussian random component to the redshift of $\sigma_{f}=150 \mathrm{~km} \mathrm{~s}^{-1}$ is added, representing an estimate of the cosmic velocity dispersion.

\subsubsection{Galaxy Properties}

Most observational maps of peculiar velocities (e.g. Willick 1991, Courteau et al. 1993, or the Sc sample) are originally drawn from 
diameter-limited catalogs such as the UGC or the ESO-Uppsala catalogs, and magnitude or other criteria are only imposed on the galaxies which already have been pre-selected by the criteria imposed in the parent catalog. In order to incorporate such selection procedures into the MC simulation, galaxies distributed according to some density field must be given diameters, magnitudes and other parameters relevant for the distance relation being used. Sodré \& Lahav (1993) have investigated the joint diameter-magnitude distribution function, and have parameterized it in the following way:

$$
\Psi(D, B) \propto \exp \left[-\frac{D}{D^{\star}}-\frac{(b-a \log (D)-B)^{2}}{2 \sigma_{M}^{2}}\right]
$$

where $B$ is the blue absolute magnitude computed from $B_{T}, D$ is the diameter of the galaxy, and the parameters $a, b, D^{\star}$ and $\sigma_{M}$ have been derived by Sodré \& Lahav. We use such a distribution to assign both diameters and blue magnitudes to the artificial galaxies. For the simulation of the Sc sample, the parameters derived by Sodré \& Lahav for their "S+I" sample with three different velocity models were averaged. The resulting parameters used are $D^{\star}=2231 \mathrm{~km} \mathrm{~s}^{-1}$ arcmin, $\mathrm{a}=-4.87, \mathrm{~b}=-0.05$.

The properties of each galaxy are assigned as follows. First the diameters in $\mathrm{km} \mathrm{s}^{-1}$ arcmin are drawn from an exponential distribution function $\propto \exp \left(D / D^{\star}\right)$ (see equation [5]). For a given diameter, we derive the most probable value for the blue absolute magnitudes $B$ from the linear relation between diameters and magnitudes, $B=a \log (D)+b$, where $a$ and $b$ are the ones from the distribution functions. The final assigned magnitude will later be computed by adding to $B$ a Gaussian random variable with a standard deviation of $\sigma_{M}$.

Since equation (5) is given in the blue band, it is necessary to relate the blue $B_{T}$ magnitude to the $I$ magnitude. For that we utilize a color-line width relation of the form $B_{T}-I=a_{c} \log w+b_{c}$. This together with the I-band $\mathrm{TF}$ relation as given in equation (1) are used to compute $I$ and line widths from $B$. Preliminary relations have been derived from the Sc data and are given in table 1. As above, the scatter in these quantities will be added in the next section.

Note that our procedure differs from that of Willick (1991), who assumes that the galaxy properties originate from a Gaussian line width distribution rather than a luminosity function. Magnitudes are then computed from the widths via a given TF relation, and a normally distributed scatter is added to the magnitudes. This results in a Gaussian luminosity function rather than the more Schechter-like function implicitly assumed in our approach.

\subsubsection{Correlations and Scatter}

In the previous section, the parameters $D$, $B, I$ and $\log w$ were assigned to each galaxy using a distribution function for $D$ and observed relations to compute the remaining parameters. Up to now, we have not considered the scatter in those relations. However, selection biases are produced because galaxy samples are selected by observables which are closely correlated with the quantity to be measured. In particular, blue magnitudes and diameters are used later to select samples. Therefore, their correlation with the parameters of the TF relation, I band magnitudes and line width, must be modeled realistically in order to obtain reliable estimates for the biases. The scatter in the relation used to 
assign $B$ from $D$ equation (5) was assumed to be $\sigma_{M}=0.709$. For the TF relation, the scatter has been estimated to be $\sigma_{T F}=0.3$. It should be emphasized that the observed scatter in the TF relation and therefore the biases depend on the line width (e.g. Giovanelli et al. 1995). The value of 0.3 chosen here is the value measured from the Sc sample at a line width $\log w$ of about 2.4. The scatter in the color - line width relation, was derived from the Sc data to be $\sigma_{c}=0.59$. In table 1 we list all the parameters used in the simulations, which are used later to determine the bias for samples of spiral galaxies. Finally, in the discussion below we also consider the uncertainty in the apparent blue magnitudes listed in the catalogs generally used to select the sample. Here we take this scatter to be $\sigma_{m}=0.3$.

Finally, we are in the position of assigning values to all of the observable quantities relevant to our simulated samples such as $B_{\text {scatter }}$, $I_{\text {scatter }}$, and $w_{\text {scatter }}$. These are computed by adding normal deviates to the corresponding quantities $B, I$ and $w$ computed before. For the inverse TF relation, four independent Gaussian random variables $\xi_{i}$ with zero mean and a $\sigma=1$ are used in the following manner. First four random variables $\sigma_{1}$ to $\sigma_{4}$ are defined:

$$
\begin{gathered}
\sigma_{1}=\sigma_{T F} \cdot \xi_{1} \\
\sigma_{2}=\sigma_{m} \cdot \xi_{2} \\
\sigma_{3}=\sqrt{\sigma_{M}^{2}-\sigma_{m}^{2}} \cdot \xi_{3} \\
\sigma_{4}=\sqrt{\sigma_{c}^{2}-\sigma_{m}^{2}} \cdot \xi_{4}
\end{gathered}
$$

These random variable are added to the the observable quantities as computed in the previous section in the following manner. First, a Gaussian with a scatter $\sigma_{M}$ has to be added to $B$ so that the distribution of $B$ and $\log D$ follows equation (5). Using the random variables defined above, we define

$$
B_{\text {scatter }}=B+\sigma_{2}+\sigma_{3}
$$

in such a way that the distribution of

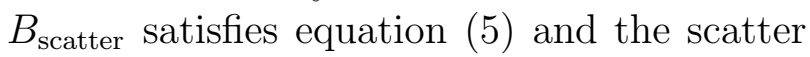
in the color relation is also reproduced if we define $I_{\text {scatter }}$ to be given by

$$
I_{\text {scatter }}=I+\sigma_{3}+\sigma_{4} .
$$

In order to reproduce the scatter in the $\mathrm{TF}$ relation, we define

$$
w_{\text {scatter }}=w \cdot 10^{\left(\sigma_{1}+\sigma_{3}+\sigma_{4}\right) / a_{T F}} .
$$

It can be verified that this leads to a Gaussian scatter term for $B-I$ with a dispersion of $\sigma_{c}$ by substituting $\sigma_{1}, \sigma_{2}$ and $\sigma_{3}$ in the relations. This is also approximately the scatter of the color - line width relation, because the additional terms added to $w$ are small compared to the scatter in the color.

Similarly, for the direct TF relation implemented by scatter in magnitudes, equations (8) to (12) change to:

$$
\begin{gathered}
\sigma_{3}=\sqrt{\sigma_{M}^{2}-\sigma_{m}^{2}-\sigma_{T F}^{2}} \cdot \xi_{3} \\
\sigma_{4}=\sqrt{\sigma_{c}^{2}-\sigma_{m}^{2}} \cdot \xi_{4} \\
B_{\text {scatter }}=B+\sigma_{1}+\sigma_{2}+\sigma_{3} \\
I_{\text {scatter }}=I+\sigma_{1}+\sigma_{3}+\sigma_{4} \\
w_{\text {scatter }}=w \cdot 10^{\left(\sigma_{3}+\sigma_{4}\right) / a_{T F}}
\end{gathered}
$$




\subsubsection{Creating Simulated Surveys}

The next step in the MC simulation is to create galaxy catalogs similar to existing ones which we assume to be complete within the specified criteria. This step is necessary to simulate the exact selection procedure of the observer. For example, a 'magnitude-limited' sample drawn from the UGC catalog is not identical to a truly magnitude limited sample because the inclusion of a galaxy in the UGC already depends on a diameter selection threshold. Since all the observables of the artificial galaxies are known, this is an easy step. For example, for the UGC we select galaxies with diameters larger than 1 arcmin or brighter than 14.5 magnitudes.

Next, "observed samples" of galaxies are drawn from the simulated catalogs. It is here that we can take full advantage of the MC approach since besides the known selection effects (e.g. on magnitudes and sizes of galaxies), even complicated selection criteria, such as imposed redshifts cutoffs, variations with direction on the sky and combination of different samples can be incorporated into our simulations. In addition, observational samples are characterized by an incompleteness function that must also be taken into account in building our simulated samples. In general, we assume that the samples are incomplete as a function of apparent blue magnitude only. The incompleteness of each sample was taken into account in generating our simulated samples.

Finally, peculiar velocities are computed from the estimated distances obtained from the $\mathrm{TF}$ relation and observed magnitudes, widths and redshifts.

\subsubsection{Computation of Bias}

The peculiar velocity of a galaxy is defined as $v_{\text {pec }}=v_{r}-d_{\text {true }}$, where $v_{r}$ includes the recessional velocity and the streaming motion at its location, and $d_{\text {true }}$ is the true velocity of the galaxy. This quantity is estimated from measurements of the redshift $c z$ and the distance $d_{\text {raw }}$ as $v_{p e c, m}=c z-d_{\text {raw }}$, where the measured redshift includes the cosmic random component $\sigma_{f}$ and the estimated distance a large measurement uncertainty. These random components lead to a bias of the measured peculiar velocity relative to $v_{p e c}$.

The final step in our MC simulation is to estimate these biases. This is done by computing raw estimates of the distances, corrected for homogeneous Malmquist bias, $d_{H M B}$ and measured redshifts $c z$ from the observed parameters, exactly in the same manner as in the observations. The error in the estimated distance $\delta_{d}$ is computed for each galaxy as

$$
-\delta_{d}=d_{H M B}-d_{\text {true }}
$$

Similarly, the error in the measured redshift relative to the underlying streaming motion and recessional velocity is computed as

$$
\delta_{z}=c z-v_{r} .
$$

The signs in these definitions were chosen so that the error in the measured peculiar velocity is $\delta_{v}=\delta_{d}+\delta_{z}$.

Biases are produced by the fact that the expectation value of these errors at a given estimated distance or measured redshift are not zero for an observed galaxy sample. In the $\mathrm{MC}$ simulation, the biases in estimated distances $b_{d}$ or in redshift space $b_{z}$ are computed by averaging the errors for individual 
galaxies in the sample within spatial cubes of $500 \mathrm{~km} \mathrm{~s}^{-1}$ size, i.e.

$$
\begin{aligned}
& \mathrm{b}_{\mathrm{d}}=<\delta_{d}>_{\text {cell }} \\
& \mathrm{b}_{\mathrm{z}}=<\delta_{z}>_{\text {cell }}
\end{aligned}
$$

Depending on whether the direct or inverse $\mathrm{TF}$ relation was used, the averaging in cells $<>_{\text {cell }}$ was performed differently. In case of the direct TF relation, galaxies were assigned to spatial cells based on the estimated distances corrected for homogeneous Malmquist bias. This results in a bias field which contains the non-trivial biases not removed by the homogeneous Malmquist correction. In case of the inverse TF relation, this assignment to cells was made in redshift space.

The average and root mean square $\sigma_{b}^{2}$ of the biases for all $N$ galaxies in each cell were estimated, homogeneous Malmquist bias corrected distances were computed and recorded. The resulting field of biases was then smoothed with a Gaussian, where the $\sigma$ in grid size was chosen to be $\left(\sigma_{b}^{2} / \sqrt{N} / 150 \mathrm{~km} \mathrm{~s}^{-1}\right)^{2 / 3}$. The minimum smoothing applied was a Gaussian with a $\sigma$ of 1 and the maximum $\sigma$ used was 3 . This results in heavy smoothing of regions with few galaxies and large discrepancies of the biases, and less smoothing in high density regions and regions where the biases of the galaxies agree well. A similar smoothing should be applied to the real data in order to make the derived bias field fully applicable.

These bias fields are later applied to real observations in the following manner. First, the raw distance estimate of real observations is corrected for homogeneous Malmquist bias. Next, this corrected distance of the galaxy and its direction on the sky is used to find the spatial cube in which the galaxy is located and the corresponding biases are determined. The distance bias $b_{d}$ is then subtracted from $d_{H M B}$ to find a final, bias corrected estimate of the distance. Similarly, the redshift bias $b_{z}$ is added to the measured redshift. The corrected distance and redshift are subsequently used to compute the peculiar velocity of the galaxy.

\section{Simulations}

In this section, we present a series of simulations of "observed" peculiar velocities distributions and compare them to the "true" velocities. The raw distances are computed from the I-band TF relation as discussed above. The simulations differ in the assumed spatial distribution of galaxies. We first present a homogeneous universe and a toy model density distribution for the purpose of demonstrating the general behavior of the bias and testing our procedure. Subsequently, we use a realistic density distribution, modeled after the density distribution derived from redshift surveys. Similar models will later be used to determine biases for existing observational samples.

\subsection{Tests of the MC Method}

We begin our analysis by applying our MC method to spatial distributions for which the expected biases can be computed analytically. This allows us to test the bias field determined from the MC method. As our first model we consider a magnitude-limited sample $\left(m_{B}=14.5\right)$ drawn from a UGC-like catalog of a homogeneous distribution of galaxies with no streaming motion. In this experiment, the peculiar velocities of the galaxies are exclusively due to the cosmic dispersion $\sigma_{f}$. Figures 1 and 2 show the biases in the 
observed peculiar velocities. In this simulation, the biases in distance $b_{d}$ are computed without the HMB correction to the estimated distances, i.e. $b_{d}=<d_{\text {raw }}-d_{\text {true }}>_{\text {cell }}$.

Figure 1 is the case corresponding to the use of the direct TF relation. Panel (a) shows the biases binned in estimated distances. Superimposed is the bias expected from a homogeneous distribution computed from equation (3). It can be seen that the bias behaves as predicted, indicating that additional selection biases are negligible. In redshift space (panel b), Malmquist-type effects result from the cosmic random component $\sigma_{f}$ and produce a bias for small redshifts. The magnitude of this bias is directly determined by the value of $150 \mathrm{~km} \mathrm{~s}^{-1}$ assumed for $\sigma_{f}$. The cosmic component plays the role of the error in distance estimate. This effect contributes to a negative bias because on the average the redshift will be underestimated. At larger redshift, this contribution becomes small and selection biases dominate.

In figure 2 we show the bias when the inverse $\mathrm{TF}$ relation is used. As before, the solid line in panel (a) represents the expected bias as estimated using now equation (4). Note that although relatively small, biases are present even for the inverse $\mathrm{TF}$ relation binned in redshift (panel b). At small redshifts, the cause is the same as in the case of the direct relation discussed above. At large redshifts, selection biases are present because of the correlation between the line width and the blue magnitudes used to select the sample due to the indirect selection on line widths introduced by the color-line width relation. This is different from the assumption of Schechter (1980) that there are no selection effects on line widths.

In our second model, we assume spherical symmetry and a radial density profile which simulates a void centered on the observer which is surrounded by a region of high density at intermediate distances. The density falls off to the mean density at large distances. The density profile is shown in figure 3. From this profile, the expected streaming motion was computed in linear approximation using $\beta=1$.

In Figure 4 and 5, the measured peculiar velocities are plotted for the direct and for the inverse TF relation, respectively. In both figures, the measured peculiar velocities and their biases are plotted as a function of estimated distances and redshifts. Again, the biases do not include the HMB correction and the analytical solutions computed from equations (3) and (4) are superimposed on the MC results.

Comparing figure 4 and 5 one can clearly see the advantage of studying the peculiar velocities in redshift space. This has previously been recognized by Willick (1994). Figure 4 shows that although selection effects are important, they are almost independent of density enhancements. This is an important property, because in this case biases do not produce any apparent infall. More importantly figure 5 shows that in the case of the inverse TF binned in redshift space, the biases are smaller than in all other combinations.

The good agreement between the analytical curves and both the homogeneous and inhomogeneous bias recovered utilizing our methodology demonstrates that our MC technique is reliable.

\subsection{Realistic Model}

In order to deal with real samples, it is necessary to apply our simulations to a density field which adequately represents the true dis- 
tribution of galaxies. As discussed above, we utilize the Optical-IRAS density field for this purpose. In contrast to the simple cases considered earlier, the bias $b_{d}$ in this section is computed from the HMB corrected distance estimates $d_{H M B}$.

The resulting velocity biases are shown in figure 6 as vectors along the line of sight at their $d_{H M B}$ positions for the case of a magnitude limited sample $(\mathrm{m}=14.5)$ and a TF relation with scatter distributed in magnitudes. For clarity, the scale of the bias vectors is five times the scale of the distances. This plot corresponds to a peculiar velocity field an observer would derive if there were no true peculiar velocities and the observer computed peculiar velocities from estimated distances corrected for HMB. It can be seen that the effect of the bias is to produce artificial infall into high-density regions. For instance this effect can be seen in the central panel near the locations of the Great Attractor $(S G X \approx-5000$, $S G Y \approx+2000)$, and the Pieces-Perseus com$\operatorname{plex}(S G X \approx+3000, S G Y \approx-3000)$.

The similarity of this map with maps of the predicted peculiar velocities from redshift surveys such as the one presented by Freudling et al. (1994) is evident. Note that these strong local biases average out if binned into homogeneous Malmquist bias corrected estimated distance bins. This is shown in Figure 7. This shows that the HMB correction works well globally, but fails locally because of the large density variations.

Above, we noted that the bias field in estimated distance space looks similar to peculiar velocity maps predicted from the OpticalIRAS model reconstruction from redshift surveys. To quantify this impression, we present in Figure 8 the apparent velocities shown in figure 6 and generated by the bias versus the velocities predicted from the Optical-IRAS model. It is such a plot which in principle can be used to derive a value for $\beta$. The comparison of the distribution of points with the lines for different $\beta$ suggests that from the data one would derive a $\beta$ in the interval $0.2-0.5$ even when the true peculiar velocities are zero, i.e. the true $\beta \approx 0$. This demonstrates that $\beta$ cannot be derived from data which have been corrected only for HMB.

In the previous section, it was argued that distances estimated with the inverse TF relation are not dominated by Malmquist bias if they are plotted as a function of redshift. Instead, such distances are biased by selection effects (see Figure 2). In order to demonstrate this difference, we plot in Figure 9 the biases for a model identical to the one shown in Figure 6 but with the scatter in the TF relation taken to be in the line width, and the biases are shown in redshift space. It can be seen that the biases show a completely different behavior than those in estimated distance space shown in Figure 6. They are mainly a function of distance, and do not concentrate around high density regions. For the chosen magnitude limited sample of 14.5, the biases are significantly smaller than the one seen in figure 6 .

\section{Applications}

While the purpose of the previous discussion was to understand the properties of Malmquist bias in combination with selection effects, the purpose of this section is to predict the magnitude of these effects for real surveys. Therefore we create simulated redshiftdistance surveys which correspond to samples actually observed. A summary of the surveys considered and their selection criteria is listed in table 2. The samples considered are 
those of Lynden-Bell et al. (1988, hereafter 7S), Willick (1990, hereafter W90) and the new Sc sample.

\subsection{S Sample}

The parameters for the simulation of the $7 \mathrm{~S}$ sample of elliptical galaxies are given in table 3 and the incompleteness as a function of magnitude was taken from Faber et al. (1989). Since the TF relation is substituted by a $D_{n}-\sigma$ relation (see table 2 ), the colorline width relation must also be replaced by the equivalent relation. We could use a relation between $D-D_{n}$ and $\sigma$, derived from the data, which plays the same role as the color-line width relation and allows for possible correlation between the parameters used to select the sample with both of the quantities of the distance relation. No significant correlation between $D-D_{n}$ and $\sigma$ was found, therefore $D_{n}$ was computed from $D$ by adding the constant and Gaussian scatter given in table 3 .

In figures 10, the derived bias field for the $7 \mathrm{~S}$ sample is shown. The biases are again shown in estimated HMB corrected distances space. The biases produce some apparent infall into the 'Great Attractor' (at $S G X \approx-4200 \mathrm{~km} \mathrm{~s}^{-1}, S G Y \approx+800 \mathrm{~km} \mathrm{~s}^{-1}$, $S G Z \approx-700 \mathrm{~km} \mathrm{~s}^{-1}$ ), but the effect is not much stronger than in other parts of the sky. In order to address the question whether such biases could produce the velocity field as suggested by $7 \mathrm{~S}$, we bin in figure 11 the biases as a function of the cosine of the angular distance from the GA in several distance shells. The binning and presentation corresponds exactly to the one used by $7 \mathrm{~S}$ in their figure 5 . It can be seen that the biases binned in such a manner are independent of the angular distance from the GA, unlike the behavior the $7 \mathrm{~S}$ find in their data. We conclude that residual biases do not significantly affect the conclusion of $7 \mathrm{~S}$ regarding the existence of a large infall towards the GA within the volume considered.

\subsection{W90 Sample}

In order to estimate the incompleteness of the W90 sample, we have binned the data in 0.5 magnitudes intervals and compared the resulting counts to those obtained in the CGCG (Zwicky et al. 1961-68) catalog. This incompleteness was imposed on the simulated sample. Subsequently, the biases were computed using the direct TF relation as discussed in section 3.3. It should be noted that the TF relation we use differs from that assumed by Willick (1990). In principle we could use the same quadratic relation, but we believe that the major source of uncertainty stems instead from the lack of detailed information regarding the properties of the scatter in the $\mathrm{TF}$ relation. In particular, the sample contains galaxies as faint as magnitude 16.5, but the number of galaxies in each magnitude interval is small. This leaves the possibility of substantial unrecognized systematic effects in the incompleteness of the sample.

In figure 12, the biases for the simulated W90 sample is shown. The figure shows the biases both as a function of redshift and as a function of $d_{H M B}$. The biases are shown separately for models with no true peculiar velocities and with a velocity field given by the Optical-IRAS model. The biases are essentially independent of the assumed velocity model. A comparison of the two panels in figure 12 shows that for a sample selected like W90, the biases out to $5000 \mathrm{~km} \mathrm{~s}^{-1}$ are smaller if the peculiar velocities are plotted as a function of $d_{H M B}$ rather than redshifts. Beyond 
that distance, the bias is similar in amplitude but with opposite sign.

In figure 13, the W90 data are corrected for the bias shown in figure 12 and binned in bins of $500 \mathrm{~km} \mathrm{~s}^{-1}$. Panel (a) corresponds to figure 1 shown by W90, whereas panels (b) and (c) show the bias corrected data as a function of redshift and distance, respectively. A significant infall into the Pisces-Perseus supercluster complex can be seen both in panels (b) and (c). While the corrections (as shown in figure 12) are substantial, the basic conclusion of W90 that there is a large bulk motion of the whole region in the direction of the Local Group cannot be ascribed to the biases discussed in this paper.

\subsection{Sc Sample}

Samples selected using a redshift criterion such as the Sc sample are a completely different case. The Sc sample also differs from the previously discussed sample by the fact that a large fraction of all galaxies which satisfy the selection criteria have actually been observed. This lowers the probability for large biases due to unrecognized systematic effects in the selection of the sample. In figure 14 the 'measured' peculiar velocities of a sample of galaxies selected with the Sc sample criteria is shown as a function of estimated distance and redshift. As before, the estimated distances in this this figure have been HMB corrected. At small distances (up to about $6000 \mathrm{~km} / \mathrm{sec}$ ) the biases in addition to the HMB are small. This reflects the changing diameter limit as a function of redshift adopted in selecting the sample. On the other hand, at large distance the peculiar velocities are strongly affected by the redshift cutoff which leads to the depopulation of galaxies with positive peculiar velocities. The full bias map which we will actually use in our analysis of the Sc sample is shown in figure 15, again plotted against HMB corrected distances.

\section{Summary}

We have presented a new approach to correct observed peculiar velocity fields. The Monte-Carlo approach we have adopted allows us to simultaneously include all known sources of biases in a realistic way. The resulting simulated biases can be applied to existing observed samples with complex but welldefined selection criteria, such as the new Sc sample.

We have tested the MC simulation in simple cases, for which bias correction schemes are known, and recovered the expected biases with high accuracy. We have also applied our technique to real samples and computed the expected biases. We found that the results presented by Lynden-Bell et al. are virtually free of biases. On the other hand, the data as presented by Willick (1990) are significantly biased, but the biases do not affect his main conclusion. Finally, we derived the expected bias for the new Sc sample of Haynes et al. (1993) that will be used in future analysis of this sample.

Acknowledgments The sample of W90 was kindly provided by Jeff Willick. GW acknowledges partial support by National Science Foundation Grant AST93-47714 and the Alexander von Humboldt Stiftung.

\section{REFERENCES}

Bothun, G.D., \& Cornell, M.E., 1990, AJ 99, 1004.

Courteau, S., Faber, S.M., Dressler, A., Willick, J.A., 1993, ApJ 412, 51. 
Dekel, A., 1993, in Observational Cosmology, ASP Conference Series Vol 51, eds G.

Chincarini, A. Iovino, T. Maccacaro and D. Maccagni, p. 194.

Dekel, A. 1994, ARAA 32, 371.

Faber, S.M., Wegner, G., Burstein, D., Davies, R.L., Dressler, A., 1989, ApJS 69, 763.

Feast, M.W. 1987, Observatory, 107, 185.

Freudling, W., da Costa, L.N., Pellegrini, P.S. 1994, MNRS, 268, 943.

Haynes, M.P., Giovanelli, R., Salzer, J.J., Wegner, G., da Costa, L.N., Freudling, W., Chamaraux, P., 1993, BAAS, 25, 1403

Giovanelli, R. et al., 1995, in preparation

Hudson,M.J., 1994, MNRS 266, 468.

Landy,S.D., \& Szalay,A.S., 1992, ApJL 394, $25 \mathrm{~L}$

Lynden-Bell, D., Faber, S.M., Burstein, D., Davies, R.L., Dressler, A., Terlevich, R.J., and Wegner, G. 1988, Ap.J., 326, 19.

Nilson,P. 1973, Uppsala General Catalog of Galaxies Uppsala Astron. Obs. Ann.6(UGC)

Schechter,P.L. 1980, ApJ 85, 801

Sodré, Jr.L. \& Lahav, O. 1993 MNRS 260, 285.

Teerikorpi, 1993, AA 280, 443.

Willick, J.A. 1991, PhD thesis, University of California at Berkeley

Willick, J.A. 1990, ApJ 351, L5.

Willick, J.A. 1994, ApJS 92, 1.

Willmer C.N.A., da Costa, L.N., Pellegrini, P.S., Fairall, A.P., Latham, D.W. \& Freudling, W., 1995, AJ 109, 61.

Zwicky,F., Herzog,E., Will,P., Karpowicz,M., Kowal,C. 1961-1968Catalog of Galaxies and
Clusters of Galaxies in six volumes California Institute of Technology, Pasadena, (CGCG) 


\section{Figure Captions}

Figure 1: Biases of an homogeneous distribution of galaxies observed with 14.5 magnitude limit drawn from the UGC catalog. The scatter of the TF relation of this sample is distributed in $\mathrm{m}$, corresponding to the direct $\mathrm{TF}$ relation. The velocities were binned in $500 \mathrm{~km} \mathrm{~s}^{-1}$ bins in estimated distance $d_{\text {raw }}$ in panel (a) and redshift $c z$ in panel (b). The units for distances, redshifts and biases are $\mathrm{km} / \mathrm{sec}$. The solid line in the panel (a) is the homogeneous Malmquist bias computed from equation (3).

Figure 2: Same figure as Figure 1, but with the scatter in the $\mathrm{TF}$ relation distributed in $\log w$, corresponding to an inverse TF relation. The solid curve in panel (a) is the expected bias for the observed distribution of galaxies computed from equation (四).

Figure 3: Density profile and peculiar velocities of the toy model described in the text.

Figure 4: Observed peculiar velocities (panels a and c) and biases (panels b and d) from the toy model for the direct TF relation. Panels (a) and (b) show the velocities and biases binned in estimated distances $d_{\text {raw }}$, whereas panels (c) and (d) show the them binned in redshift. The dashed lines in panels (a) and (b) are the expected homogeneous Malmquist bias. The solid curve in panel (b) is the bias as computed from equation (3), using the known density profile as shown in figure 3. Note the strong bias for the redshift binning (panel d).

Figure 5: Observed peculiar velocities and biases from the toy model for the inverse TF relation. The presentation is the same is in figure 4. The solid curve in panel (b) is the bias as computed from equation (4), using the observed distribution of galaxies derived from the data. Note that there is some bias even for the redshift binning (panel d).

Figure 6: Biases of a 14.5 magnitude limited sample shown as a function of HMB corrected positions. The biases were computed using the direct TF relation and are shown as vectors at their positions in Supergalactic Cartesian coordinates. This plot corresponds to a peculiar velocity field an observer would derive if there were no true peculiar velocities and the observer computed peculiar velocities from estimated distances corrected for HMB. The units for distances are $\mathrm{km} / \mathrm{sec}$. The scale of the velocity vectors is five times the scale of distances.

Figure 7: The biases shown in figure 6 binned in HMB corrected estimated distances bins. Note the very small amplitude of the bias if averaged over the whole sky.

Figure 8: Estimated HMB corrected peculiar velocities for a model without true peculiar velocities plotted versus the peculiar velocity as predicted by the Optical-IRAS model for $\beta=1$. If no biases were present, the two quantities should not correlate. The observed correlation between estimated and predicted velocities could be mistaken as evidence for gravitationally induced streaming motion if biases are not taken into account. The lines show expected correlations for several different values of $\beta$. The observed correlation is similar to the one expected for values of $\beta$ between 0.2 and 0.5 .

Figure 9: Biases of distances derived from the TF relation for a 14.5 magnitude limited sample shown in redshift space. The bias map shown here is again plotted as a function of Supergalactic coordinates. The velocity vectors are five times the scale of distances. This is to be compared to figure 6 , which shows a similar plot shown in corrected estimated dis- 
tance space, which has a completely different behavior of the biases.

Figure 10: Simulated biases for the 7S sample, binned in estimated distance space. The scale of the arrows is 5 times the scale of distances.

Figure 11: The unsmoothed biases from figure 10 plotted as a function of the cosine of the angular distance from the Great Attractor in 3 estimated distance ranges, similar to figure 5 in $7 \mathrm{~S}$. It can be seen that conclusions by $7 \mathrm{~S}$ based on this plot are not subject to biases.

Figure 12: Biases computed for the simulated sample of Willick (1990). The panel (a) shows the biases as a function of redshift. Panel (b) shows the biases as a function of HMB corrected distances. The error bars show the biases computed for a model with a velocity field as described in the text. The solid points show the biases for a model without any 'true' velocities. The solid points are offset horizontally from their true position by $100 \mathrm{~km} \mathrm{~s}^{-1}$ in order to make them easier to distinguish from the error bars.

Figure 13: The W90 sample corrected for biases and binned in bins of $500 \mathrm{~km} \mathrm{~s}^{-1}$. Panel (a) shows the raw data as a function of redshift in the CMB restframe. Panel (b) shows the corrected peculiar velocities binned in redshift bins of $500 \mathrm{~km} \mathrm{~s}^{-1}$ size. Panel (c) shows the corrected data binned in equivalent estimated distance bins.

Figure 14: HMB corrected estimates of the peculiar velocities of a sample of galaxies selected like the Sc sample, binned in estimated distances (panel a) and redshifts (panel b). The small points indicate individual galaxies, whereas the large points are averages in bins of $500 \mathrm{~km} \mathrm{~s}^{-1}$. The dashed line is the computed homogeneous Malmquist bias. The most prominent feature when plotted as a function of the estimated distance is the redshift cutoff at $7500 \mathrm{~km} \mathrm{~s}^{-1}$, which has the effect that galaxies with a redshift $>7500$ $\mathrm{km} \mathrm{s}^{-1}$ are only included if they have negative peculiar velocities.

Figure 15: Bias map for the simulated sample of Haynes et al. (1994). The unit of the velocity vectors is identical to the unit of distances. The vectors are plotted at the position of the estimated distances corrected for homogeneous Malmquist bias. 
Table 2: Selection Criteria of Samples

\begin{tabular}{ccccccc} 
Sample & catalogs & type & area & mag. & sizes & redshifts \\
\hline Sc & UGC & Sc & $|b|>10^{\circ}$ & & $5^{\prime}>d>2.5^{\prime}$ & $<3000$ \\
& ESO & & & $5^{\prime}>d>1.5^{\prime}$ & 3000 to 5000 \\
& MGC & & & $5^{\prime}>d>1.3^{\prime}$ & 5000 to 7500 \\
\hline $7 \mathrm{~S}$ & $\mathrm{RC} 2$ & $\mathrm{E}$ & $|b|>2.5^{\circ}$ & $B_{T}^{o}<13.0$ & $d>2^{\prime}$ & $<8000$ \\
& $\mathrm{RSA}$ & & & & \\
& $\mathrm{UGC}$ & & & & \\
& $\mathrm{ESO}$ & & & & \\
\hline Willick & $\mathrm{UGC}$ & Spirals & $70<l<160^{\circ}$ & $<16.5$ & $d>0.6^{\prime}$ & \\
& $\mathrm{CGCG}$ & & $b<0$ & & & \\
\hline
\end{tabular}


Table 1: Model Parameters for Spiral galaxies

\begin{tabular}{cr}
\hline Luminosity & function \\
\hline$D^{*} \mathrm{~km} \mathrm{~s}^{-1}$ & 2231 \\
$\mathrm{a}$ & -4.87 \\
$\mathrm{~b}$ & -0.05 \\
$\sigma_{M}$ & 0.709 \\
\hline $\mathrm{TF}$ & relation \\
\hline$\sigma_{T F}$ & 0.3 \\
$a_{T F}$ & -7.48 \\
$b_{T F}$ & -2.53 \\
\hline$\sigma_{m}$ & 0.3 \\
\hline$a_{c}$ & 1.78 \\
$b_{c}$ & -2.54 \\
$\sigma_{c}$ & 0.59
\end{tabular}

Table 3: Model Parameters for $\mathrm{E}+\mathrm{S} 0$

\begin{tabular}{cr}
\hline Luminosity & function \\
\hline$D^{*} \mathrm{~km} \mathrm{~s}^{-1}$ & 2489 \\
$\mathrm{a}$ & -4.62 \\
$\mathrm{~b}$ & -1.66 \\
$\sigma_{M}$ & 0.396 \\
\hline$D_{n}-\sigma$ & relation \\
\hline$\sigma_{D n-\sigma}$ & 0.6 \\
$a_{D n-\sigma}$ & 1.2 \\
\hline$\sigma_{m}$ & 0.3 \\
\hline$D-D_{n}$ & 0.05 \\
$\sigma_{D-D_{n}}$ & 0.5
\end{tabular}


Figure 1: Biases of an homogeneous distribution of galaxies observed with 14.5 magnitude limit drawn from the UGC catalog. The scatter of the TF relation of this sample is distributed in $\mathrm{m}$, corresponding to the direct $\mathrm{TF}$ relation. The velocities were binned in $500 \mathrm{~km} \mathrm{~s}^{-1}$ bins in estimated distance $d_{\text {raw }}$ in panel (a) and redshift $c z$ in panel (b). The units for distances, redshifts and biases are $\mathrm{km} / \mathrm{sec}$. The solid line in the panel (a) is the homogeneous Malmquist bias computed from equation (3). 
Figure 2: Same figure as Figure 1, but with the scatter in the TF relation distributed in $\log w$, corresponding to an inverse TF relation. The solid curve in panel (a) is the expected bias for the observed distribution of galaxies computed from equation (4). 
Figure 3: Density profile and peculiar velocities of the toy model described in the text. 
Figure 4: Observed peculiar velocities (panels a and c) and biases (panels b and d) from the toy model for the direct TF relation. Panels (a) and (b) show the velocities and biases binned in estimated distances $d_{\text {raw }}$, whereas panels (c) and (d) show the them binned in redshift. The dashed lines in panels (a) and (b) are the expected homogeneous Malmquist bias. The solid curve in panel (b) is the bias as computed from equation (3), using the known density profile as shown in figure 3. Note the strong bias for the redshift binning (panel d). 
Figure 5: Observed peculiar velocities and biases from the toy model for the inverse TF relation. The presentation is the same is in figure 4. The solid curve in panel (b) is the bias as computed from equation (4), using the observed distribution of galaxies derived from the data. Note that there is some bias even for the redshift binning (panel d). 
Figure 6: Biases of a 14.5 magnitude limited sample shown as a function of HMB corrected positions. The biases were computed using the direct TF relation and are shown as vectors at their positions in Supergalactic Cartesian coordinates. This plot corresponds to a peculiar velocity field an observer would derive if there were no true peculiar velocities and the observer computed peculiar velocities from estimated distances corrected for HMB. The units for distances are $\mathrm{km} / \mathrm{sec}$. The scale of the velocity vectors is five times the scale of distances. 
Figure 7: The biases shown in figure 6 binned in HMB corrected estimated distances bins. Note the very small amplitude of the bias if averaged over the whole sky. 
Figure 8: Estimated HMB corrected peculiar velocities for a model without true peculiar velocities plotted versus the peculiar velocity as predicted by the Optical-IRAS model for $\beta=1$. If no biases were present, the two quantities should not correlate. The observed correlation between estimated and predicted velocities could be mistaken as evidence for gravitationally induced streaming motion if biases are not taken into account. The lines show expected correlations for several different values of $\beta$. The observed correlation is similar to the one expected for values of $\beta$ between 0.2 and 0.5 . 
Figure 9: Biases of distances derived from the TF relation for a 14.5 magnitude limited sample shown in redshift space. The bias map shown here is again plotted as a function of Supergalactic coordinates. The velocity vectors are five times the scale of distances. This is to be compared to figure 6 , which shows a similar plot shown in corrected estimated distance space, which has a completely different behavior of the biases. 
Figure 10: Simulated biases for the 7S sample, binned in estimated distance space. The scale of the arrows is 5 times the scale of distances. 
Figure 11: The unsmoothed biases from figure 10 plotted as a function of the cosine of the angular distance from the Great Attractor in 3 estimated distance ranges, similar to figure 5 in $7 \mathrm{~S}$. It can be seen that conclusions by $7 \mathrm{~S}$ based on this plot are not subject to biases. 
Figure 12: Biases computed for the simulated sample of Willick (1990). The panel (a) shows the biases as a function of redshift. Panel (b) shows the biases as a function of HMB corrected distances. The error bars show the biases computed for a model with a velocity field as described in the text. The solid points show the biases for a model without any 'true' velocities. The solid points are offset horizontally from their true position by $100 \mathrm{~km} \mathrm{~s}^{-1}$ in order to make them easier to distinguish from the error bars. 
Figure 13: The W90 sample corrected for biases and binned in bins of $500 \mathrm{~km} \mathrm{~s}^{-1}$. Panel (a) shows the raw data as a function of redshift in the CMB restframe. Panel (b) shows the corrected peculiar velocities binned in redshift bins of $500 \mathrm{~km} \mathrm{~s}^{-1}$ size. Panel (c) shows the corrected data binned in equivalent estimated distance bins. 
Figure 14: HMB corrected estimates of the peculiar velocities of a sample of galaxies selected like the Sc sample, binned in estimated distances (panel a) and redshifts (panel b). The small points indicate individual galaxies, whereas the large points are averages in bins of $500 \mathrm{~km} \mathrm{~s}^{-1}$. The dashed line is the computed homogeneous Malmquist bias. The most prominent feature when plotted as a function of the estimated distance is the redshift cutoff at $7500 \mathrm{~km} \mathrm{~s}^{-1}$, which has the effect that galaxies with a redshift $>7500 \mathrm{~km} \mathrm{~s}^{-1}$ are only included if they have negative peculiar velocities. 
Figure 15: Bias map for the simulated sample of Haynes et al. (1994). The unit of the velocity vectors is identical to the unit of distances. The vectors are plotted at the position of the estimated distances corrected for homogeneous Malmquist bias. 